\title{
Investigating the Involvement of Cytoskeletal Proteins MreB and FtsZ in the Origin of Legume-Rhizobial Symbiosis
}

\author{
Wenlong Zhao, ${ }^{1}$ Huixia Zhu, ${ }^{1}$ Feng Wei, ${ }^{1}$ Donglai Zhou, ${ }^{1}$ Youguo $\mathrm{Li}^{1}{ }^{1 \dagger}$ and Xue-Xian Zhang ${ }^{2}$ \\ ${ }^{1}$ State Key Laboratory of Agricultural Microbiology, Huazhong Agricultural University, Wuhan 430070, People's Republic of \\ China \\ ${ }^{2}$ School of Natural and Computational Sciences, Massey University, Auckland 0745, New Zealand
}

Accepted 15 February 2021.

\begin{abstract}
Rhizobia are rod-shaped bacteria that form nitrogen-fixing root nodules on leguminous plants; however, they don't carry MreB, a key determinant of rod-like cell shape. Here, we introduced an actin-like mreB homolog from a pseudomonad into Mesorhizobium huakuii 7653R (a microsymbiont of Astragalus sinicus L.) and examined the molecular, cellular, and symbiotic phenotypes of the resultant mutant. Exogenous mreB caused an enlarged cell size and slower growth in laboratory medium. However, the mutant formed small, ineffective nodules on A. sinicus $\left(\mathrm{Nod}^{+} \mathrm{Fix}^{-}\right.$), and rhizobial cells in the infection zone were unable to differentiate into bacteroids. RNA sequencing analysis also revealed minor effects of $m r e B$ on global gene expression in free-living cells but larger effects for cells grown in planta. Differentially expressed nodule-specific genes include cell cycle regulators such as the tubulin-like $f t s Z_{1}$ and $f t s Z_{2}$. Unlike the ubiquitous $\mathrm{FtsZ}_{1}$, an $\mathrm{FtsZ}_{2}$ homolog was commonly found in Rhizobium, Sinorhizobium, and Mesorhizobium spp. but not in closely related nonsymbiotic species. Bacterial twohybrid analysis revealed that MreB interacts with FtsZ $_{1}$ and FtsZ $_{2}$, which are targeted by the host-derived nodule-specific cysteine-rich peptides. Significantly, MreB mutation D283A disrupted the protein-protein interactions and restored the aforementioned phenotypic defects caused by MreB in M. huakuii. Together, our data indicate that MreB is detrimental for modern rhizobia and its interaction with $\mathrm{FtsZ}_{1}$ and $\mathrm{FtsZ}_{2}$ causes the symbiotic process to cease at the late stage of bacteroid differentiation. These findings led to a hypothesis that loss of $m r e B$ in the common ancestor of members of Rhizobiales and subsequent acquisition of $f t s Z_{2}$ are critical evolutionary steps leading to legume-rhizobial symbiosis.
\end{abstract}

Keywords: bacteria-plant symbiosis, bacteroid, cell shape, FtsZ, MreB, Rhizobium-legume symbiosis

${ }^{\dagger}$ Corresponding author: Y. Li; youguoli@mail.hzau.edu.cn

Funding: This research was financially supported by funds from the $\mathrm{Na}$ tional Key Research and Development Program of China (grant number 2019YFA09004700) and the National Natural Science Foundation of China (grant numbers 31772401 and 31970267). X.-X. Zhang acknowledges support from the New Zealand MBIE Catalyst Fund (project number 92846082 ).

*The e-Xtra logo stands for "electronic extra" and indicates that supplementary materials are published online.

The author(s) declare no conflict of interest.
Cytoskeletons are of fundamental importance for all cellular organisms, because they function as key regulators of many cellular processes such as the determination of cell shape, division, DNA segregation, polarity, motility, and intracellular trafficking of macromolecules. Eukaryotic cells have long been known to possess three major types of cytoskeletal filaments: microtubules (hollow tube-like polymers of tubulins), actin microfilaments, and intermediate filaments (Carballido-López 2006; Shih and Rothfield 2006). However, it was approximately only 30 years ago that their structural and functional homologs were discovered in prokaryotic cells, including tubulin-like FtsZ (RayChaudhuri and Park 1992), actin-like MreB (Wachi et al. 1987), and the intermediate filament-like crescentin (Charbon et al. 2009). Since then, bacterial cytoskeletal proteins have received continuous research attention. Although many critical molecular details are still lacking, it is now known in general how these cytoskeletons determine cell shape and mediate cell division. However, we still have a very limited understanding of why bacteria have evolved diverse cytoskeletal systems and how they contribute to the ecological success of various bacterial species in their natural environments (Caccamo and Brun 2018; Faguy and Doolittle 1998; Shi et al. 2018; Szwedziak and Löwe 2013).

The shape of a bacterial cell is largely determined by the cell wall structure, particularly the formation of peptidoglycan (PG) networks during the process of bacterial growth (Egan et al. 2020; Typas et al. 2011). Most bacterial species are rod shaped and show a cylindrical cellular structure with a relatively stable diameter but a variable longitudinal axis along which most of the growth takes place. The FtsZ tubulin is a developmental regulator, which polymerizes into rings at the cell-division sites and drives cytokinesis. On the other hand, the actin-like MreB polymers bind to the cytoplasmic face of the inner membrane and coordinate activities of the PG synthesis enzymes, which eventually determines the shape and size of the cell and also the mode of cellular elongation. Cell division and cell wall synthesis are coupled by the direct protein-protein interactions between FtsZ and MreB. Critical MreB residues involved in the FtsZ interactions have recently been identified in Escherichia coli (Asp-285) and also in Caulobacter crescentus (Asp-12 and Glu-141) (Dye et al. 2011; Fenton and Gerdes 2013).

Although FtsZ is widely conserved in bacteria, MreB is limited to rod-like and curved bacterial species. Depletion of mreB expression in E. coli, C. crescentus, and Pseudomonas aeruginosa resulted in spherical cells that were nonviable when grown in standard laboratory media (Kruse et al. 2005; Robertson et al. 2007; Shi et al. 2017; Takacs et al. 2010). Notably, previous phylogenetic studies further suggested that modern spherical cells were most likely evolved from rod-shaped ancestors via the loss of the $m r e B$ gene (Daniel and Errington 2003; Siefert and Fox 1998; Yulo and Hendrickson 2019). Loss and regaining 
of $m r e B$ may have occurred multiple times through separate events of horizontal gene transfers (Morita and Nishida 2018).

MreB is well established to be the key determinant of rod-like cell shape (Errington 2015; Shi et al. 2018) but there are exceptions: species of Actinobacteria and Rhizobiales are rod shaped but lack MreB and other components of the elongase complex such as MreC, MreD, and RodZ (Margolin 2009). The MreBlacking cells grow by polar extension rather than homogenous enlargement along the longitudinal axis, as observed in MreBcontaining cells (Brown et al. 2012; Daniel and Errington 2003). The order Rhizobiales contains many species that form mutualistic or pathogenic associations with plants and animals. A typical example concerns rhizobia that infect the roots of leguminous plants, leading to the formation of specific, highly differentiated plant organs called nodules (Masson-Boivin and Sachs 2018; Poole et al. 2018). Each nodule contains hundreds to thousands of symbiotic plant cells which are each infected with thousands of nitrogen-fixing rhizobial cells. Within the intermediate nodules formed in symbioses such as Sinorhizobium meliloti-Medicago sativa L. (alfalfa) and Mesorhizobium huakuii-Astragalus sinicus L. (Chinese milk vetch), rhizobial cells lose their original rod shape and undergo terminal differentiation into bacteroids. The enlarged bacteroid cells display variable shapes and they are polyploid, containing 16 to 32 copies of the genome (Mergaert et al. 2006).

Bacteroid differentiation is complex. It involves the master regulator CtrA, whose activities influence the expression of over 462 genes associated with the cell cycle (De Nisco et al. 2014). The cell differentiation process is partially driven by the plantderived nodule-specific cysteine-rich (NCR) peptides, and requires the functional bacteroid development factor $A$ (bacA) gene encoding a peptide transporter in $S$. meliloti and also in $M$. huakuii (LeVier and Walker 2001; Penterman et al. 2014; Tan et al. 2009). NCR peptides target multiple cellular processes in rhizobia, including direct interactions with tubulin-like FtsZ proteins (Farkas et al. 2014; Van de Velde et al. 2010). Considering the fact that rhizobial cells lack MreB and possess a symbiotic lifestyle with bacteroid differentiation, it is reasonable to hypothesize that loss of MreB is likely a critical step for the evolutionary origin of Rhizobium-legume symbiosis.

Here, we describe an empirical test of the abovementioned hypothesis by introducing an exogenous $m r e B$ gene into $M$. huakuii 7653R followed by molecular, cellular, and symbiotic characterization of the resulting mutant cells. Our work began with the cloning of $m r e B$ from a model organism of $P$. fluorescens SBW25 (Zhang and Rainey 2013). Expression of $m r e B$ caused morphological changes of $M$. huakuii free-living cells and severe defects in symbiosis with the A. sinicus host plant. This led to further investigations into the underlying molecular and cellular mechanisms using the methods of bacterial two-hybrid analysis and high-throughput RNA sequencing (RNA-seq) in combination with light and electron microscopy analysis. Our combined data indicate that exogenous MreB directly interacts with rhizobial FtsZ homologs and disrupts the normal process of bacteroid differentiation and nodule development. Phylogenetic analysis indicate that rhizobial $f t s Z_{2}$ genes in the Rhizobiaceae and Phyllobacteraceae families formed a separate cluster distinct from the ubiquitous $f t s Z_{l}$. Finally, we identified the host-derived NCR peptides that specifically target Fts $\mathrm{Z}_{1}$ and $\mathrm{Fts}_{2}$ in $M$. huakuii 7653R. On the basis of the these findings, the potential roles of MreB will be discussed together with FtsZ in regard to the evolutionary origin of Rhizobium-legume symbiosis.

\section{RESULTS}

\section{Exogenous mreB caused larger cell size and} slower growth in vitro.

The $m r e B$ gene (pflu0863) of $P$. fluorescens SBW25 was cloned into the plasmid vector pBBR1MCS5, then introduced into M. huakuii 7653R. The resultant strain 7653R-mreB was first subject to cellular visualization under light and scanning electron microscopes. Representative images are shown in Figure 1A and B, respectively. The mutant 7653R-mreB cells remained rod shaped but appeared to have longer cellular length when compared with wild-type $7653 \mathrm{R}$ and a mutant containing the plasmid vector (i.e., negative control). Next, we measured cell length and width for 50 cells each on the basis of the scanning electron microscope images. Results confirmed the finding that exogenous $m r e B$ caused a significant increase of cellular volume in terms of length/width ratios (Supplementary Table S1). When growth kinetics were measured in tryptone yeast (TY) broth medium, the 7653RmreB mutant displayed a clear growth defect when compared with the vector-only control 7653R-pBBR (Fig. 1C). Cell synchronization followed by flow cytometry analysis showed that the 7653R-mreB mutant contained more DNA than the wild-type cells (Supplementary Fig. S1).

Having shown the cellular effects of $m r e B$, we then asked whether the exogenous MreB functions in a similar manner as previously described in other model organisms. To this end, an MreB-enhanced green fluorescent protein (eGFP) fusion was constructed in the genetic background of $M$. huakuii 7653R, and the resulting mutant was subjected to fluorescent microscopy analysis. Indeed, the MreB-eGFP protein was likely to be arranged in a spiral structure (Fig. 1C). This directly shows that the foreign MreB was successfully expressed in M. huakuii, and it was somehow involved in the cell wall synthesis.

\section{Exogeneous mreB produced deleterious effects on the symbiotic performance of $M$. huakuii.}

Plant experiments were performed with A. sinicus seedlings inoculated separately with three rhizobial strains (7653R, 7653R-mreB, and the vector-only control 7653RpBBR). At 28 days after inoculation, plants were harvested and subjected to symbiotic characterization with regards to the standard parameters. Like the uninoculated control, plants inoculated with 7653R-MreB showed the typical signs of nitrogen deficiency (i.e., smaller plants with yellowish leaves) (Fig. 2A), and a significant reduction of shoot fresh weight was observed (Fig. 2C). The 7653R-mreB mutant produced significantly less root nodule in terms of both nodule number and nodule weight per plant, and the related nodules showed very little nitrogenase activity (Fig. 2C). Next, internal structures of the nodules were examined under a microscope using the method of paraffin-embedded tissue sectioning (Fig. 2B). Nodules formed by wild-type 7653R were filled with highly differentiated bacteroids. In contrast, nodules of the 7653R-mreB mutant contained fewer rhizobial cells in the infection zones. Finally, we used a fluorescent microscope to examine the nodules formed by $g f p$-labeled variants of $7653 \mathrm{R}$ and its mutant 7653R-mreB. Parallel to our expectation, the nodules differed significantly in the abundance of rhizobial cells (marked with green color) (Fig. 2D). Although the 7653R-mreB nodule did contain rhizobial cells in the infection zone, it is very likely that exogenous $m r e B$ caused severe defects in the late stage of root nodule development and cellular differentiation into nitrogen-fixing bacteroids.

\section{Determining the molecular interactions between MreB and FtsZ.}

Having shown that exogenous $m r e B$ causes significant phenotypic changes of $M$. huakuii, we proceeded to unravel the underlying molecular mechanisms. First, we tested the hypothesis that MreB produces the effects via protein-protein interactions with FtsZ. The MreB-FtsZ interactions are crucial for determining the rod shape of model organisms such as E. coli, C. crescentus, 
and Bacillus subtilis. More importantly, critical MreB residues involved in the protein-protein interactions had been identified. These include Asp-285 in E. coli MG1655 (Fenton and Gerdes 2013) and Asp-10 and Glu-140 in C. crescentus (Dye et al. 2011). The genome of 7653R contains two fts $Z$ homologs ( fts $Z_{1}$ and $\left.f t s Z_{2}\right)$, which are encoded by MCHK_RS15005 and MCHK_RS03685 and show 41.71 and $31.31 \%$ amino acid sequence identity, respectively, with FtsZ from E. coli MG1655. Therefore, the BacterioMatch II two-hybrid system was used to examine the potential in vivo interactions between FtsZ (FtsZ or $\mathrm{FtsZ}_{2}$ ) and $\mathrm{MreB}\left(\mathrm{MreB}^{\mathrm{WT}}, \mathrm{MreB}^{\mathrm{D} 283 \mathrm{~A}}\right.$, or $\left.\mathrm{MreB}^{\mathrm{D} 12 \mathrm{~A}-\mathrm{E} 141 \mathrm{~A}}\right)$ (details below). Results are summarized in Figure 3. Strong interactions were detected for wild-type MreB with both FtsZ $_{1}$ and $\mathrm{FtsZ}_{2}$, and the activities were severely disrupted by mutation D283A in MreB. Moderate effects were observed for the MreB ${ }^{\mathrm{D} 12 \mathrm{~A}-\mathrm{E} 141 \mathrm{~A}}$ variant.
The MreB-FtsZ interaction interferes with Rhizobium-legume symbiosis.

To further assess the symbiotic significance of the MreB-FtsZ interaction, we constructed two rhizobial mutants (7653R-mreB1 and 7653R-mreB2) which carry an $m r e B$ allele with the abovementioned $\mathrm{MreB}^{\mathrm{D} 283 \mathrm{~A}}$ and $\mathrm{MreB}^{\mathrm{D} 12 \mathrm{~A}-\mathrm{E} 141 \mathrm{~A}}$ mutations, respectively. Together with 7653R and 7653R-MreB, their symbiotic phenotypes were assessed on $A$. sinicus (Fig. 4). The MreB ${ }^{\text {D12A-E141A }}$ mutant (strain 7653R-mreB2) showed defects similar to those of the wildtype $m r e B$ strain (7653R-MreB), whereas the 7653R-mreB1 was comparable with wild-type 7653R in terms of nodule morphology (Fig. 4A) and nitrogenase activities (Fig. 4B). Paraffin section experiments clearly showed that nodules formed by $7653 \mathrm{R}-\mathrm{mreB} 1$ had the normal internal structures (Fig. 4A).

Furthermore, we examined bacteroid differentiation using live or dead microscopy analysis (Fig. 5). Like wild-type 7653R,
A
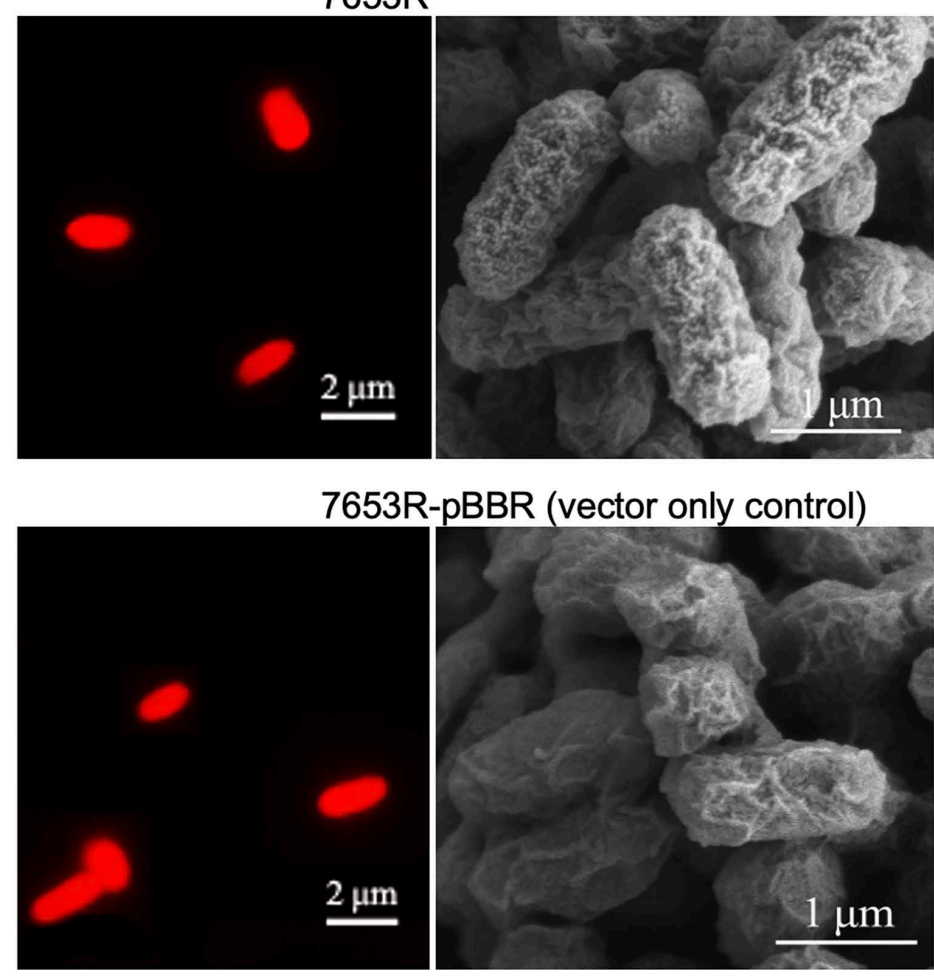

R-pBR (vector only control)

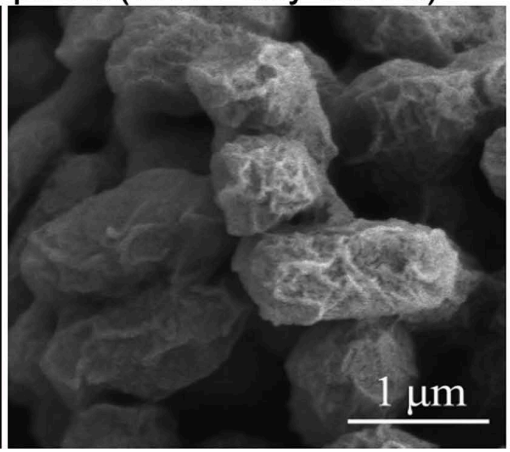

7653R-mreB
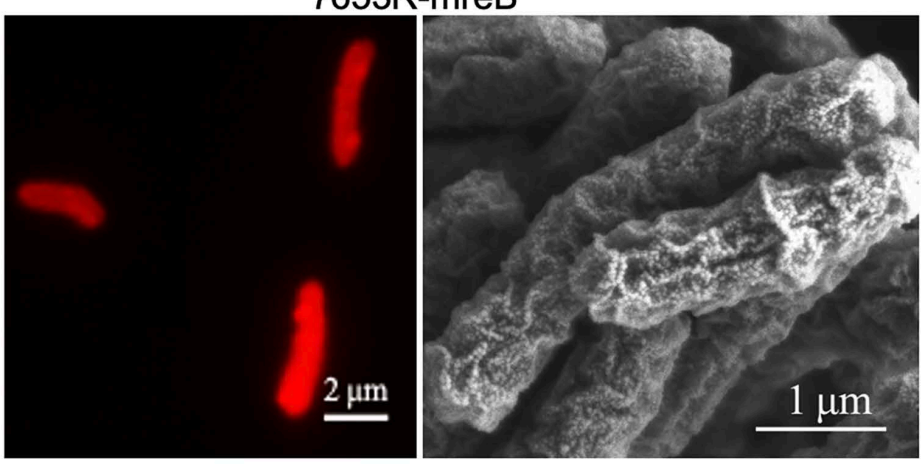

B
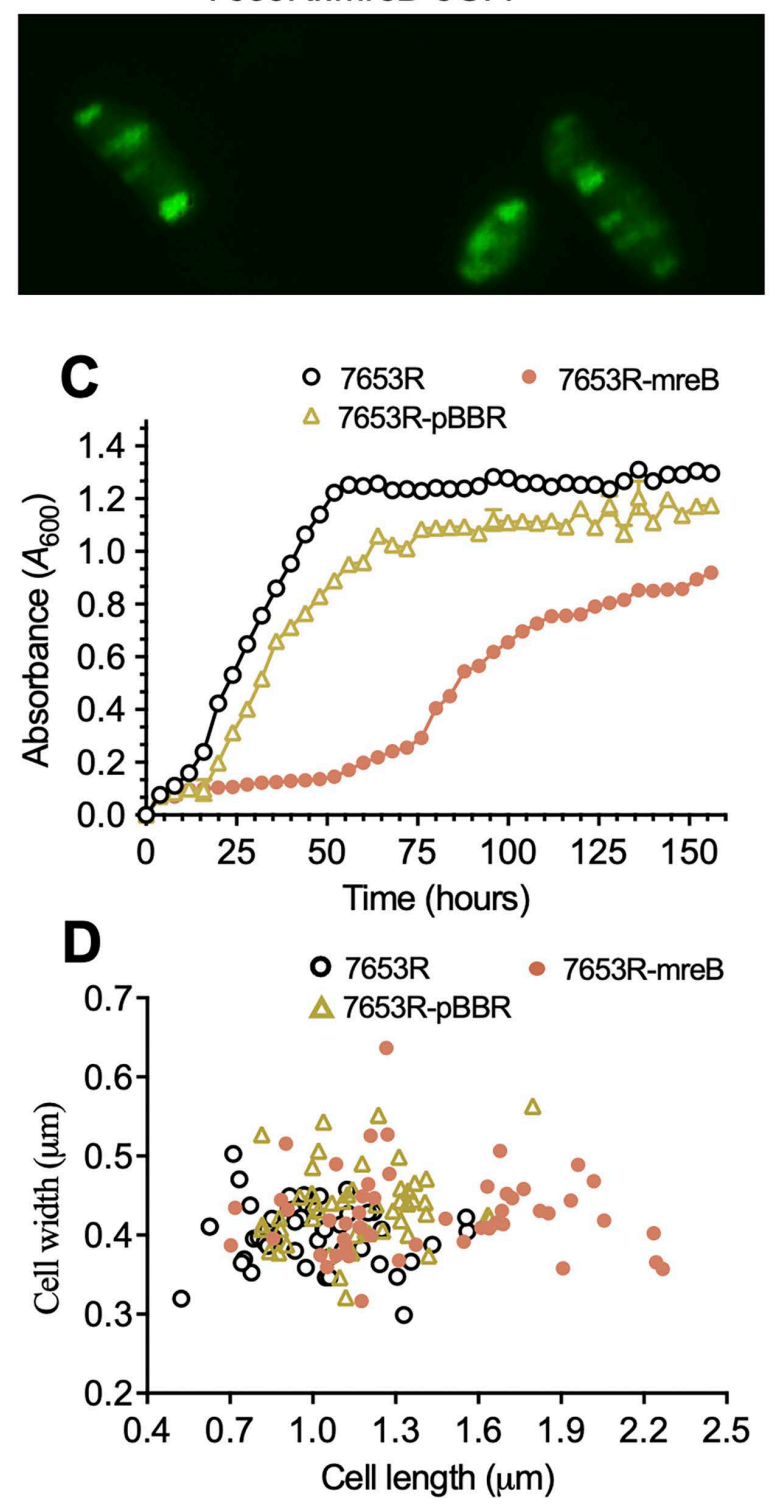

Fig. 1. Phenotypic characterization of Mesorhizobium huakuii harboring exogenous mreB in free-living conditions. A, Cell images under fluorescence microscope (left panel) and scanning electron microscope (SEM) (right panel). B, Fluorescence microcopy image showing cellular location of the MreB-enhanced green fluorescent protein (eGFP) fusion expressed in the wild-type genetic background. C, Growth dynamics of the three strains in tryptone yeast broth. In this particular assay, gentamycin was added for 7653R-pBBR and 7653R-mreB to help maintain the plasmid. This explains the slightly slower growth of 7653R-pBBR compared with the wild type. $A_{600}=$ absorbance at $600 \mathrm{~nm}$. D, Size distribution of bacterial cells measured using SEM images. $7653 \mathrm{R}$, wild type; 7653R-pBBR, negative control with the plasmid vector only; 7653R-mreB, a 7653R-derived mutant containing mreB from Pseudomonas fluorescens. 
mutant 7653R-mreB1 formed nodules filled with well-differentiated bacteroid cells. In contrast, nodules formed by 7653RMreB and 7653R-mreB2 contained a small amount of living rhizobial cells. Many bacteroid-like cells were stained red as dead cells. It is interesting to note that all three mutants showed similar cell shapes with a slower growth phenotype in laboratory medium (data not shown). Together, our data consistently indicate that exogenous $m r e B$ interferes with bacteroid differentiation via interactions with $\mathrm{FtsZ}_{1}$ and $\mathrm{FtsZ}_{2}$.

\section{$\mathrm{FtsZ}_{2}$ is phylogenetically distinct from $\mathrm{FtsZ}_{1}$.}

Next, we examined the distribution and phylogeny of FtsZ homologs in the genomes of 36 representative species in the Rhizobiales order (Supplementary Table S2). The resultant neighborjoining tree is shown in Figure 6. Gene $f t s Z_{1}$ was ubiquitously found in all genomes, whereas $f t s Z_{2}$ was restricted to certain plant-associated species, including species of Sinorhizobium, Mesorhizbium, Bradyrhizbium, and the Rhizobium/Agrobacterium group that form nitrogen-fixing root nodules on legumes. Azorhizobium caulinodans ORS571 was an exception, and it contains only one copy of ftsZ. However, A. caulinodans ORS571 is capable of fixing nitrogen in aerobic free-living conditions and forms nodules on the roots and also on the stem of the tropical legume Sesbania rostrata. It is interesting to note that the two nonsymbiotic species of Mesorhizbium don't carry $f_{t s} Z_{2}$. M. terrae was isolated from soil and $M$. oceanicum B7 represents a group of oval- shaped bacteria isolated from deep seawater (Fu et al. 2017). Importantly, fts $Z_{2}$ genes of Rhizobium, Sinorhizobium, and Mesorhizobium spp. formed a distinct cluster well separated from the related $\mathrm{FtsZ}_{1}$ homologs (Fig. 6), suggesting that $f t s Z_{2}$ was laterally acquired before divergence into different families or genera. However, the Bradyrhizobium fts $Z_{2}$ likely originated from a different source, because it was closely related to $f t s Z_{1}$ genes in the Bradyrhizobium cluster. Together, the phylogenetic data suggest that the evolution of $f t s Z$, particularly the acquisition of $f t s Z_{2}$, was involved in the origin of legume-rhizobial symbiosis, at least for rhizobia in the Rhizobiaceae and Phyllobacteraceae families.

\section{RNA-seq revealed global effects of exogenous $m r e B$} on rhizobial gene expression.

Finally, we sought to determine the influences of exogenous $m r e B$ on gene expression on a global scale. To this end, RNAseq was performed using RNA samples prepared from 7653R and 7653R-mreB cells grown in TY laboratory medium and root nodules. Cluster analysis showed that the patterns of differentially expressed genes (DEGs) were primarily determined by the growth conditions (in vitro versus in vivo) and then by the genotypes (wild type versus mutant) (Fig. 7A).

When grown in laboratory medium, 347 DEGs were identified between the two strains, among which 103 genes were upregulated and 244 downregulated (Fig. 7B). The upregulated genes in 7653R-mreB are dominated by transporters and include $16 \mathrm{~S}$ and
A
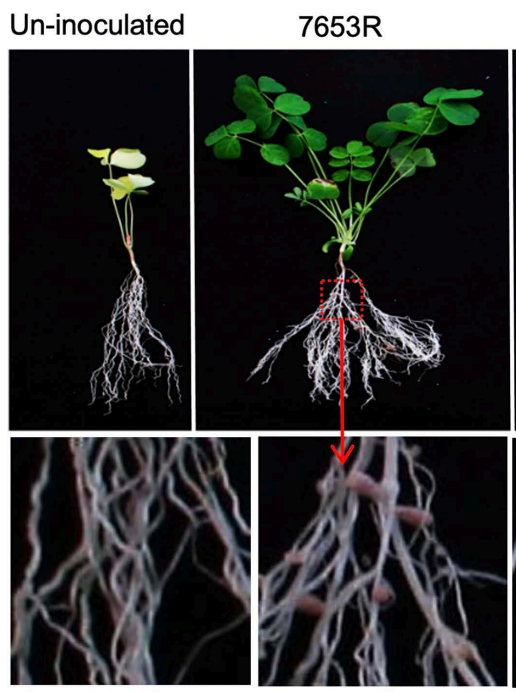

C

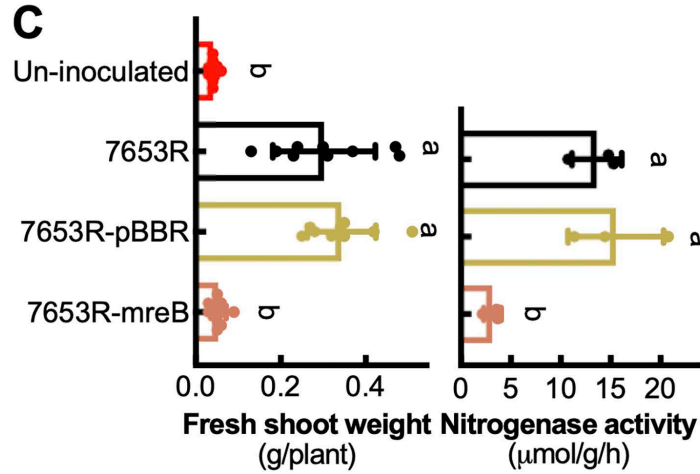

7653R-pBBR
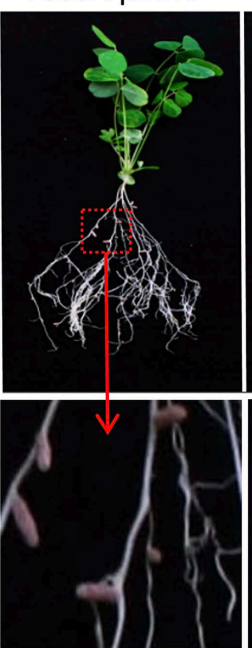

7653R-mreB

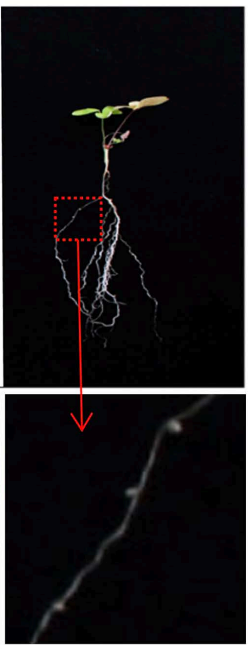

B

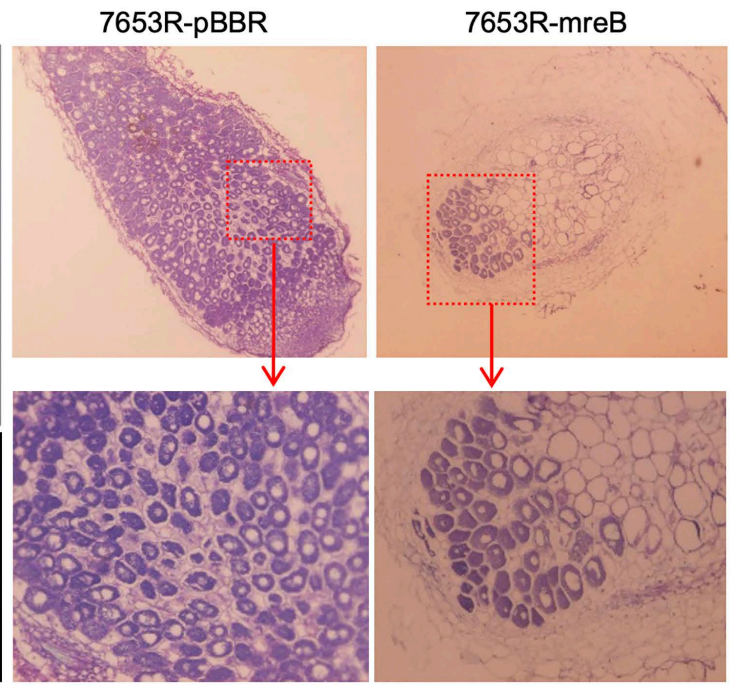

D

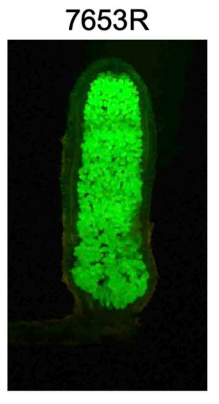

7653R-mreB

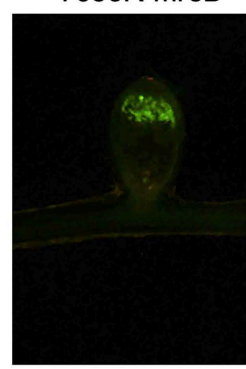

Fig. 2. Symbiotic phenotypes of Mesorhizobium huakuii harboring exogenous mreB. A, Astragalus sinicus plants inoculated with wild-type 7653R and two isogenic mutants with $m r e B$ or vector only as a control (7653R-pBRR). B, Paraffin-embedded sections of root nodules stained with toluidine blue. C, Symbiotic properties of the three rhizobial strains on A. sinicus. Data are means and standard errors of three to nine biological replicates. Bars that are not connected by the same letter (shown above each) are significantly different $(P<0.05)$ by one-way analysis of variance multiple comparisons. D, Representative images of fluorescence microscopy analysis of bacteroids within nodules. The two rhizobial strains were labeled with enhanced green fluorescent protein. 
23S ribosomal RNA genes (Supplementary Table S3). Most of the genes expressed in the wild type with more than 100-fold of fragments per kilobase per millions mapped reads but not expressed in the mutant are all located in the plasmids associated with symbiosis (Supplementary Table S4). Gene ontology (GO) term enrichment analysis revealed that DEGs were mostly involved in the biological process and molecular function (Fig. 7D).

In the nodule, 745 DEGs were identified in the 7653R-mreB mutant, among which 327 were upregulated and 418 downregulated (Fig. 7B). GO term enrichment analysis revealed that the downregulated genes were mostly associated with molecular function in symbiosis, including transition metal ion binding and iron-sulfur cluster assembly (Fig. 7C). Most of these DEGs are related to nif genes for nitrogen fixation (e.g., nifH encoding the Fe protein component of the nitrogenase and nifDK encoding the $\alpha$ and $\beta$ subunits of the Mo-Fe protein).

Next, protein-protein interaction (PPI) network analysis was performed for DEGs identified in both free-living cells and bacteroids. Most genes in the network showed the same trend of up- or downregulation. However, there are some exceptions. For example, glmS, rpoN, hypD, hypE, fold1, nifQ, and nifT were upregulated in free-living cells but downregulated in nodules; and the opposite was found for fumC, $r p s N, \operatorname{gmh} B, \operatorname{aldH}, k d s B$, leuD, typA, hisB, kamA, $m f D$, and ugpC (Supplementary Table S5). CtrA is a key regulator for cell cycle and bacteroid differentiation in M. huakuii (Peng et al. 2014). When the PPI subnetwork was constructed for DEGs in the CtrA regulon, significant changes between the wild type and mutant $7653 \mathrm{R}$-mreB were detected only in nodules but not in free-living cells (Supplementary Fig. S2). Similarly, slight changes was observed only in nodules for DEGs associated with the cell cycle (Supplementary Table S6).

\section{Validation of nodule-specific DEGs}

by quantitative reverse-transcription PCR.

Five genes of our interests were selected for validation by quantitative reverse-transcription (qRT)-PCR using total RNA

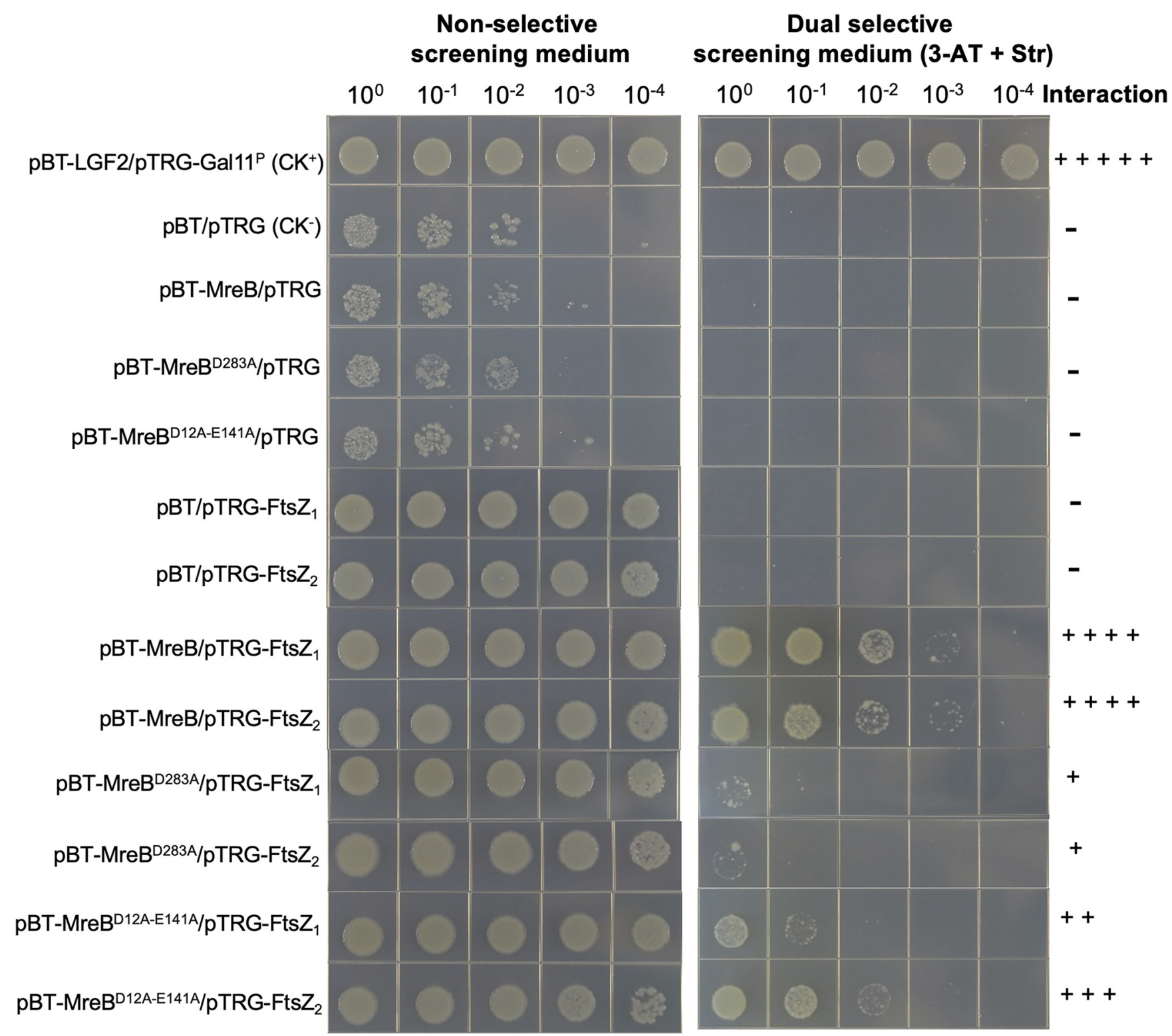

Fig. 3. Bacterial two-hybrid assays showing the direct interactions of MreB with Fts $Z_{1}$ and Fts $Z_{2}$. Wild-type $m r e B$ and the two mutant alleles $\left(\mathrm{MreB}^{\mathrm{D} 283 \mathrm{~A}}\right.$ and $\mathrm{MreB}{ }^{\mathrm{D} 12 \mathrm{~A}-\mathrm{E} 141 \mathrm{~A}}$ ) were separately cloned into plasmid pBT, whereas fts $Z_{1}$ and fts $Z_{2}$ genes were cloned into the pTRG vector. Escherichia coli strains containing various pairs of pBT and pTRG recombinant plasmids were grown in parallel on nonselective screening media and selective screening media containing 3-amino-1,2,4-triazole (3-AT) $(5 \mathrm{mM})$ and streptomycin (Str). Assays included a positive control (CK $\left.{ }^{+}\right)$also a negative control $\left(\mathrm{CK}^{-}\right)$provided in the BacterioMatch II Two-Hybrid System (Agilent Technologies, Inc.). 
samples prepared from free-living cells and bacteroids in the nodules (Fig. 8). BacA is a membrane-integral pyrophosphorylase, which participates in peptidoglycan biosynthesis and determines bacitracin susceptibility. BacA function is essentially required for bacteroid differentiation in rhizobia, including $M$. huakuii. Gene bacA was expressed at elevated levels within nodules and exogenous $m r e B$ caused a threefold increase (Fig. 8A). No significant difference was observed for gene ctrA when bacteria were grown both in vitro and in vivo (Fig. 8B). The actin-like FtsA was expressed at relatively higher levels with the introduction of $m r e B$, and the same was observed for both freeliving and bacteroid cells (Fig. 8C). Intriguingly, expression of $f t s Z_{1}$ was significantly reduced in bacteroids but such a reduction was not observed in the 7653R-mreB mutant (Fig. 8D). In contrast, $m r e B$ caused $f t s Z_{2}$ to be expressed at the highest level in nodules (Fig. 8E).

\section{Interactions between $\mathrm{FtsZ}_{1}$ and $\mathrm{FtsZ}_{2}$ with host-derived NCR peptides.}

Finally, we tested the hypothesis that exogenous MreB interferes the potential interactions of FtsZ with NCR peptides, which are known, in general, to play key regulatory roles in bacteroid differentiation. To this end, the bacterial two-hybrid system was employed to examine the potential direct interactions in vivo between $\mathrm{FtsZ}_{1}$ and $\mathrm{FtsZ}_{2}$ and a panel of nine NCR peptides encoded in the genome of $A$. sinicus. The results are summarized in Supplementary Figure S3. Protein-protein interactions were observed for $\mathrm{FtsZ}_{2}$ with five of the nine NCR peptides (namely, NCR160, NCR154, NCR126, NCR101, and NCR083), and a weak interaction with NCR026. Only one NCR (NCR167) was able to interact with $\mathrm{FtsZ}_{1}$. Intriguingly, NCR167 targeted FtsZ 1 but not $\mathrm{FtsZ}_{2}$, indicating distinct functions of $\mathrm{FtsZ}_{1}$ and $\mathrm{FtsZ}_{2}$ in bacteroid differentiation.

\section{DISCUSSION}

The Rhizobium-legume symbiosis has been one of the bestunderstood examples of mutualism between microorganisms and their eukaryotic hosts (Haag and Mergaert 2020; Poole et al. 2018). The symbiotic process involves sequential exchange of signaling molecules (Oldroyd et al. 2011). Briefly, plant-derived flavonoids cause specific production of nodulation factors by rhizobia, which is then recognized by the plant, leading to production of NCR peptides. These guide the differentiation of rhizobial cells into bacteroids, coupled with plant differentiation into root nodules. Importantly, symbiotic nitrogen fixation plays an important role in global nitrogen cycling (Kang et al. 2020). Thus, an interesting question in biology has been how such a fascinating symbiotic relationship was first evolved approximately 100 million years ago (Coba de la Peña et al. 2018; Marchetti et al. 2014; Masson-Boivin and Sachs 2018; Wang et al. 2020; Zhang and Rainey 2010).

Data presented here suggest that loss of the actin-like MreB is likely involved in the ancient symbiotic innovation between rhizobia and leguminous plants. This hypothesis is mainly based on previous phylogenetic analysis suggesting that rod is the ancestral cell shape of modern bacteria (Siefert and Fox 1998), and the fact that rhizobial symbionts have evolved the unique MreB-independent mechanisms for maintaining rod shape (Brown et al. 2012). It is likely that the MreB-dependent cell morphologies and the associated modes of bacterial growth were not suitable for the plantregulated process of intracellular rhizobial differentiation into bacteroids. Thus, loss of MreB in the common ancestor of Rhizobiales is a crucial step for the origin and evolution of Rhizobium-legume symbiosis. This hypothesis is generally supported by the empirical data presented here with regard to the influences of an exogenous $m r e B$ as revealed in the $M$. huakuii-A. sinicus model system.
A

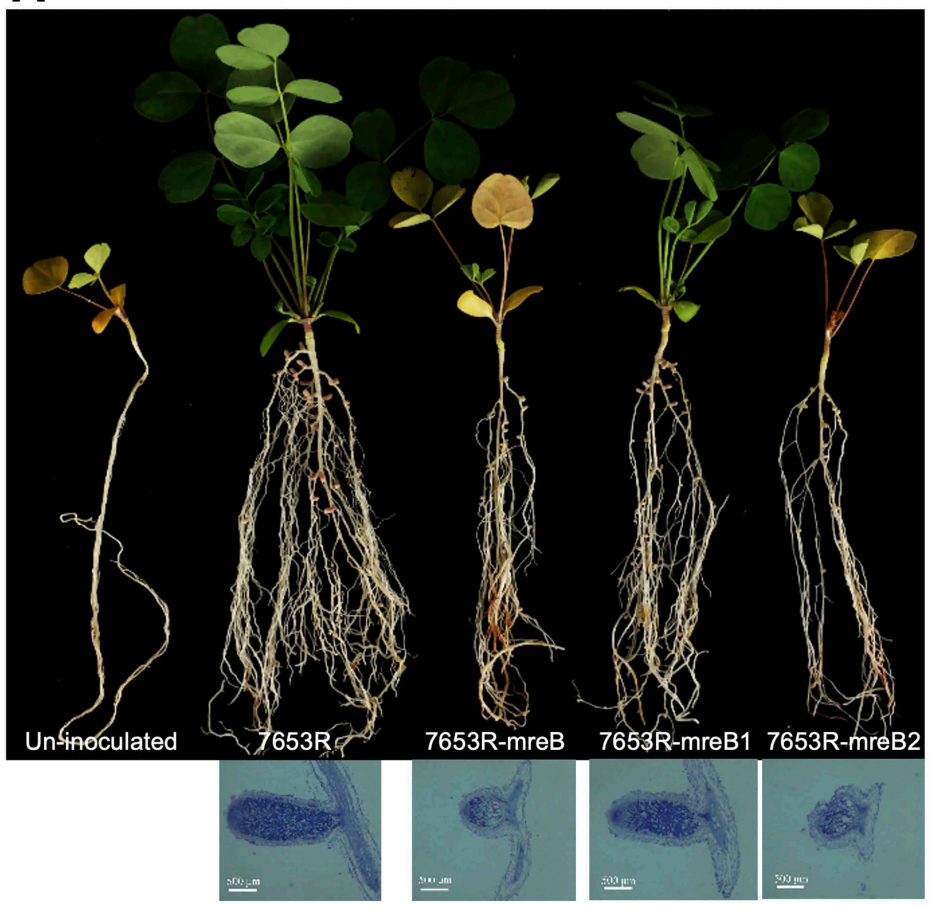

B
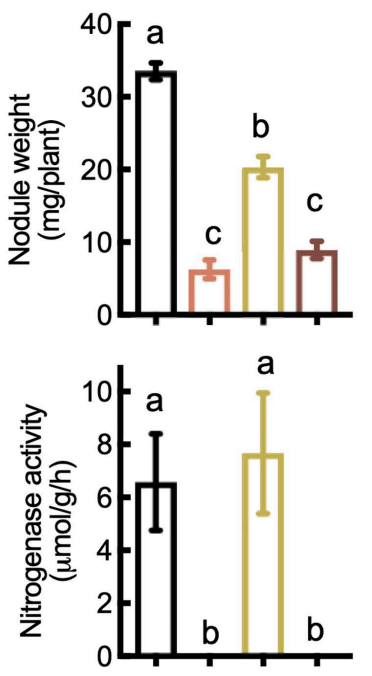

$7653 R$

Un-inoculated
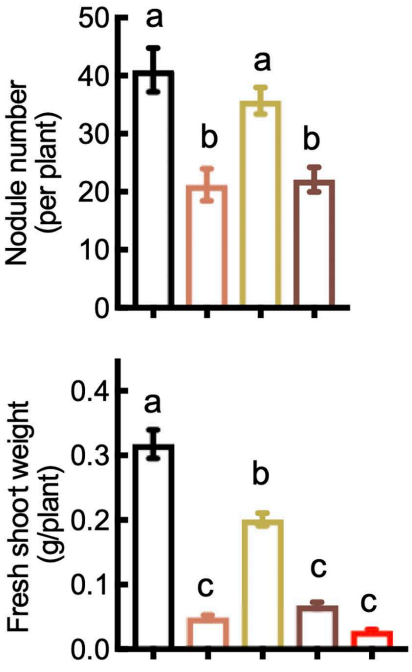

7653R-mreB (MreB ${ }^{\mathrm{WT}}$ )

7653R-mreB1 (MreB $\left.{ }^{\mathrm{D} 283 \mathrm{~A}}\right)$

Fig. 4. Symbiotic properties of Mesorhizobium huakuii carrying the two mreB mutant alleles. A, Astragalus sinicus plants showing the effects of inoculation with wild-type $7653 \mathrm{R}$ and its derived mutants. Representative images of nodule sections stained with toluidine blue are provided at the bottom. B, Symbiotic performance was assessed in terms of nodule weight, nodule number, nitrogenase activity, and fresh shoot weight. Data are means and standard errors of three to nine biological replicates. Bars that are not connected by the same letter (shown above each) are significantly different $(P<0.05)$ by one-way analysis of variance multiple comparisons. 
First, we showed that the $m r e B$ gene from $P$. fluorescens SBW25 was functionally expressed in the genetic background of $M$. huakuii 7653R. Results of the MreB-eGFP fusion clearly indicate that the exogenous MreB was capable of integrating into the cell envelope structure (Fig. 1B), most likely in discrete and disconnected patches expected from the current MreB working models (Errington 2015). This finding was initially surprising, because $P$. fluorescens SBW25 belongs to class Gammaproteobacteria whereas M. huakuii 7653R is a member of the Alphaproteobacteria class. A recent large-scale comparative genomic analysis suggested that Rhizobiales originated as free-living ancestor approximately 1,500 million years ago. Thus, our data suggest that MreB (and its interaction with FtsZ) is functionally conserved at least between the two distinct classes within the Proteobacteria phylum, despite the long-term adaptation of rhizobia to a lifestyle without MreB.

Second, our phenotypic data consistently indicate that exogenous MreB has relatively minor effects on free-living bacterial growth in laboratory media; on the contrary, more severe defects were observed for the $m r e B$-containing mutant during symbiosis with the A. sinicus host. The symbiotic process can be divided into two main stages: (i) early invasion leading to the formation of nodules and (ii) the late-stage of nodule development, including the differentiation of nitrogen-fixing bacteroids within nodule cells (Haag and Mergaert 2020; Masson-Boivin and Sachs
2018). Although exogenous $m r e B$ resulted in enlarged and slowgrowing rhizobial cells, the effects on the early stage of symbiosis were minimal and the mutant 7653R-mreB remained $\mathrm{Nod}^{+}$ (Fig. 2). However, further development of the nodule and differentiation of rhizobial cells into functional bacteroids ceased at the second stage of the symbiosis (Fix ${ }^{-}$). The symbiotic phenotypes were further supported by the global patterns of gene expression for rhizobial cells grown in vitro and in vivo. Genes with predicted functions in cell cycle and bacteroid development were among the identified DEGs whose expression was altered by the exogenous $m r e B$ gene.

At the molecular level, we show that the detrimental effects observed for the exogenous MreB were mediated almost solely through its direct interactions with $\mathrm{FtsZ}_{1}$ or $\mathrm{FtsZ}_{2}$. Of particular note is that the MreB mutation of D283A completely abolished the in vivo protein-protein interactions between $\mathrm{MreB}$ and $\mathrm{FtsZ}_{1}$ (or Fts $Z_{2}$ ), as revealed by the bacterial two-hybrid assays. Moreover, when the corresponding $m r e B$ variant was introduced into $M$. huakuii 7653R, the resultant mutant (7653R-mreB1) displayed similar phenotypes both in laboratory media and in planta. As in S. meliloti, the genome of M. huakuii 7653R possesses two copies of fts $Z$ homologs. Our unpublished data showed that both $f t s Z_{1}$ and $f t s Z_{2}$ are functionally required for the formation of nitrogen-fixing nodules in the roots of $A$. sinicus. Here, we identified the NCR peptides that specifically target

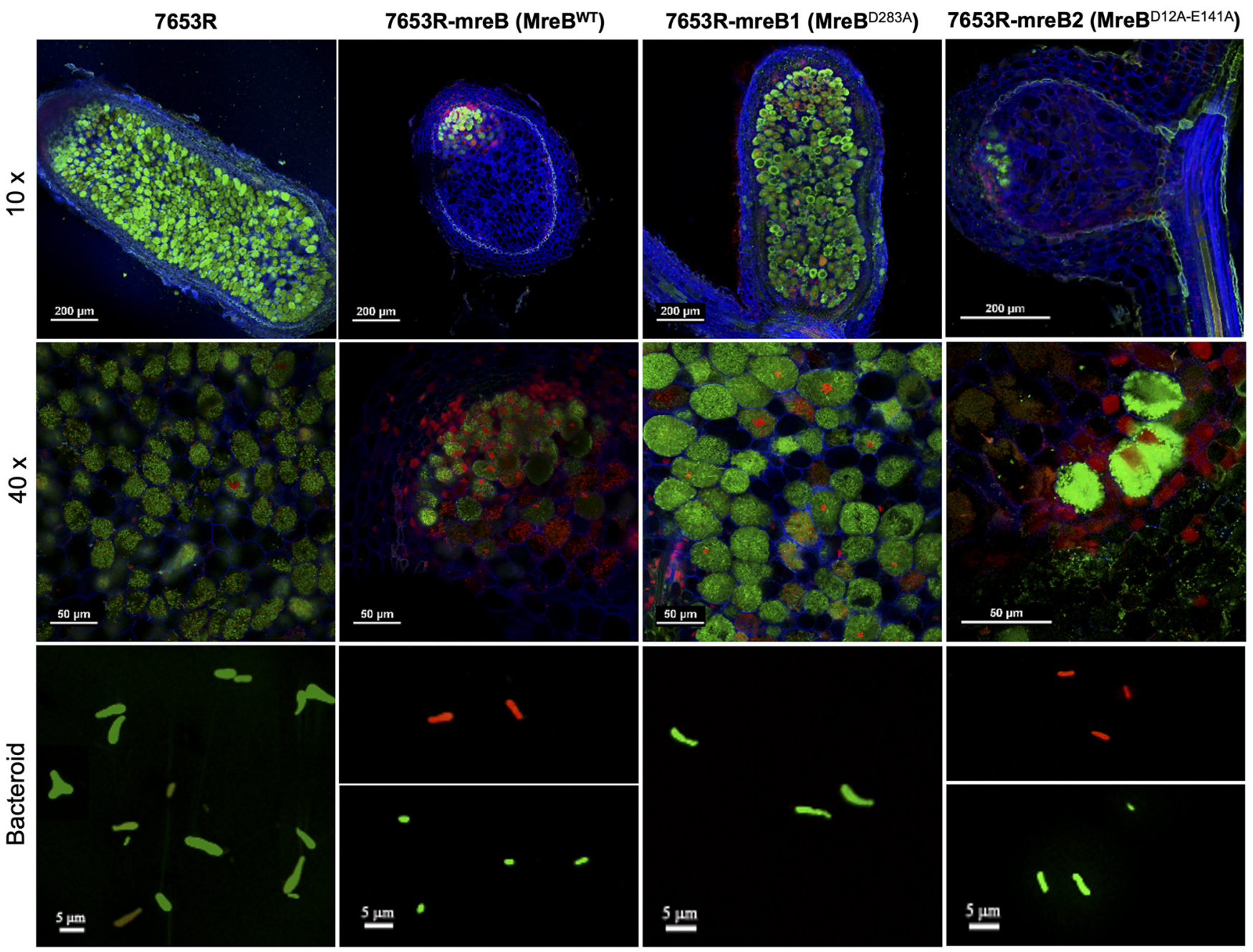

Fig. 5. Effects of exogenous $m r e B$ on bacteroid differentiation. Live or dead staining was performed with three fluorescent dyes using root nodules of Astragalus sinicus harvested on the 28th day after inoculation. Viable and dead bacterial cells were stained green and red by SYTO9 and propidium iodide, respectively. Plant cells were stained blue by M2R. Representative images with a $\times 10$ and $\times 40$ objective lens and a close visualization of the bacteroid-like cells are shown in three panels for each of the four rhizobial strains. 
FtsZ $_{1}$ and $\mathrm{FtsZ}_{2}$. The plant-derived NCR peptides are key regulators that govern the rhizobial process of bacteroid differentiation. Therefore, it makes complete sense that the exogenous MreB interferes with $\mathrm{FtsZ}_{1}$ and $\mathrm{FtsZ}_{2}$ functions, causing the symbiotic process to cease at the late nitrogen-fixing stage.

Finally, we sought to understand why rhizobial strains harbor two ftsZ homologs in their genomes. A common feature of FtsZ (or $\mathrm{FtsZ}_{1}$ in the case of two copies) in Rhizobiales is a
C-terminal extension found in certain members of Alphaproteobacteria, which is missing in $\mathrm{FtsZ}_{2}$ homologs. Thus, it is reasonable to speculate that rhizobial $\mathrm{FtsZ}_{1}$ originated from the ancestral Rhizobiales, whereas $\mathrm{FtsZ}_{2}$ was later acquired as a result of symbiotic adaptation to plants (Fig. 6). It appears that genera Azorhizobium and Bradyrhizobium had divergent routes from other rhizobial genera (i.e., Rhizobium, Sinorhizobium, and Mesorhizobium). A. caulinodans ORS571 possesses only one

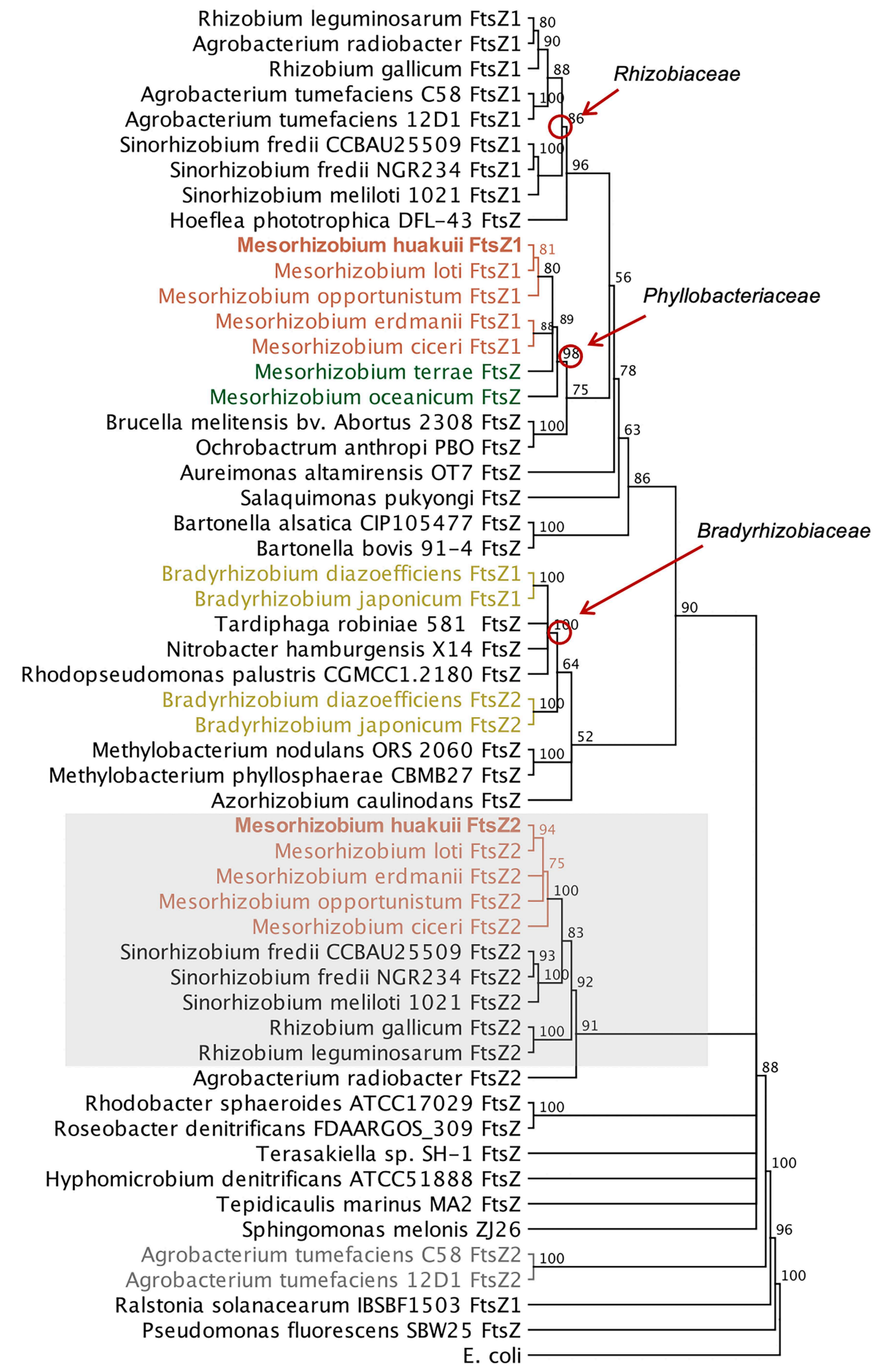

Fig. 6. Phylogenetic tree of FtsZ homologs in different families of nitrogen-fixing rhizobia and their related nonsymbiotic bacteria. For strains with two copies of FtsZ, the larger one is named FtsZ $Z_{1}$ whereas the smaller one is called FtsZ $Z_{2}$. The rhizobial FtsZ $Z_{2}$ cluster is marked with gray. 
FtsZ, and the two copies of FtsZ are closely related for Bradyrhizobium spp. Interestingly, it has been recently proposed that the first nodulating lineage arose from either Azorhizobium or Bradyrhizobium approximately 150 to 80 million years ago, after the emergence of legumes on earth (Wang et al. 2020). For symbiotic nitrogen-fixing members of Rhizobium, Sinorhizobium, and Mesorhizobium, they may share a common origin of $f t s Z_{2}$, whose functions are essential for legumerhizobial symbiosis. Rhizobia need to maintain cellular integrity in both free-living and symbiotic statuses. Thus, it is highly possible that certain cell division genes are dedicated for specific roles in maintaining cell envelope structures either in vitro or in vivo, or during the transition of life style from free-living in soil to bacteroid within a root nodule. In
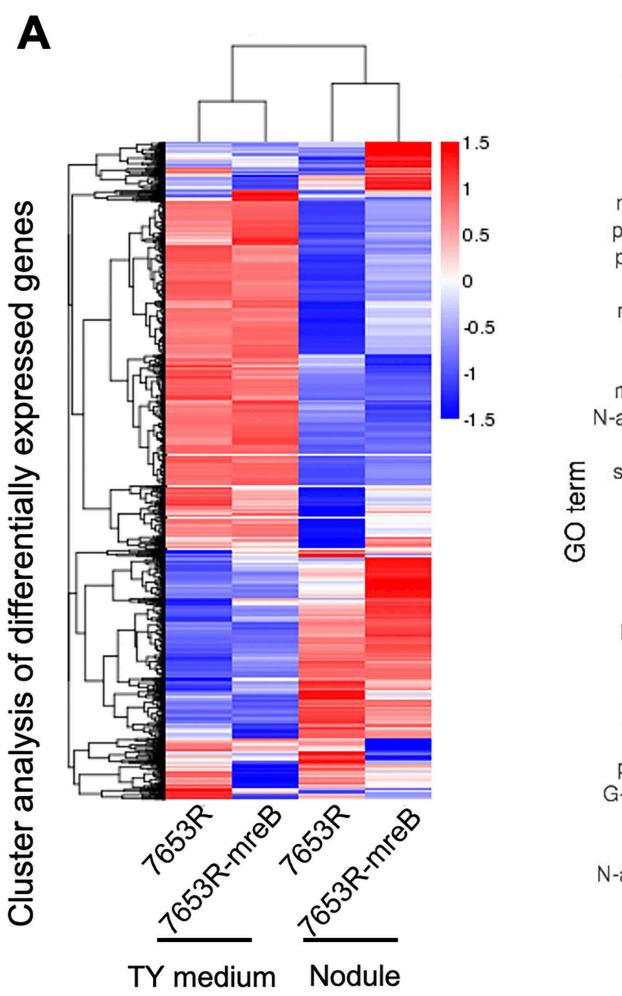

C
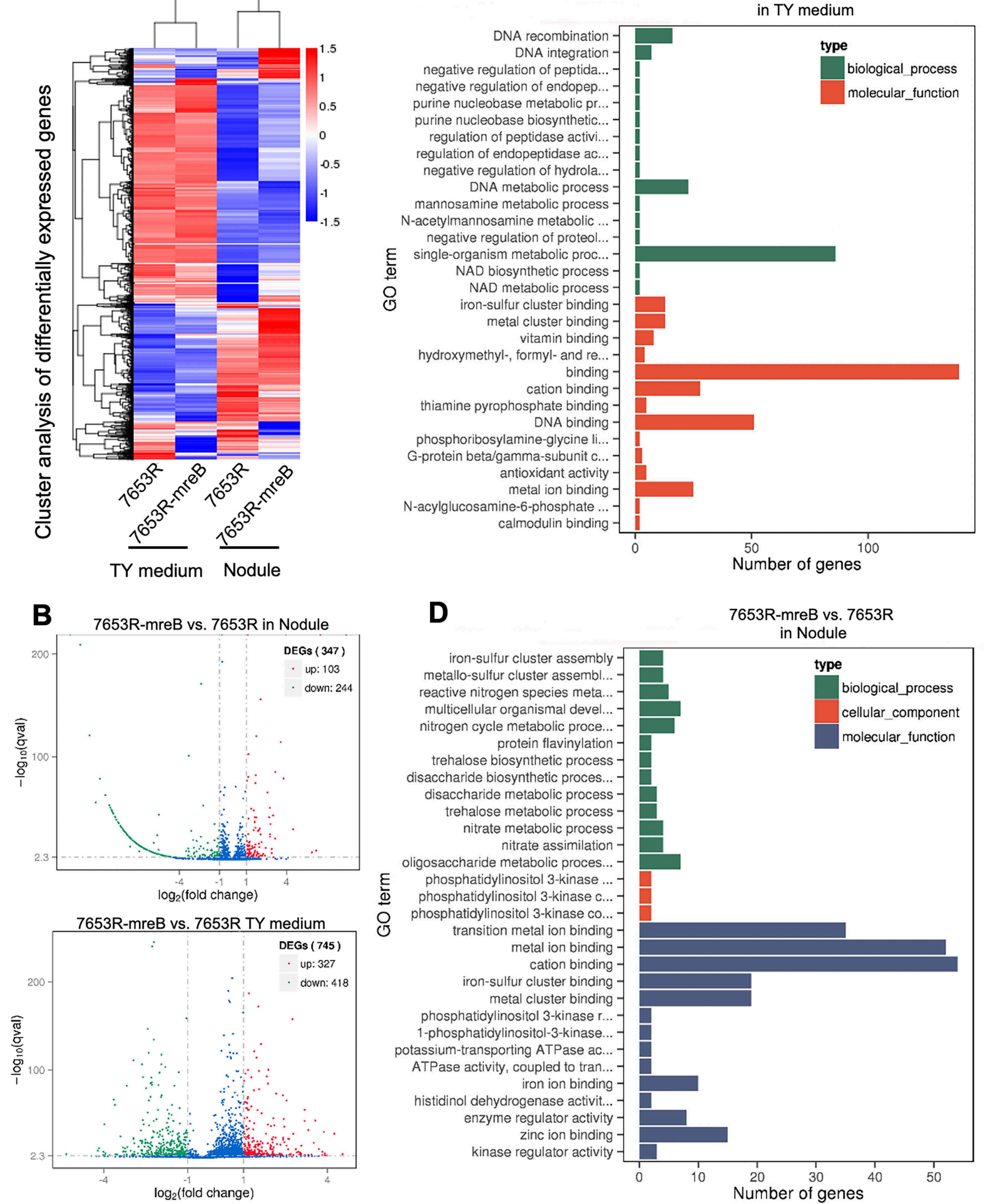

Fig. 7. Effects of exogenous mreB on global gene expression for free-living and symbiotic rhizobial cells. A, Cluster analysis of differentially expressed genes (DEGs) for 7653R and 7653R-mreB grown in tryptone yeast (TY) medium and the nodule of Astragalus sinicus. B, Volcano plots showing DEGs that were upregulated (red) or downregulated in 7653R-mreB mutant relative to wild-type 7653R. C, Gene ontology (GO) term enrichment analysis of DEGs identified in free-living cells. D, GO term enrichment analysis of DEGs identified for rhizobial cells in nodules. 
S. meliloti, $f t s Z_{2}$ appears to be essential for free-living growth (Margolin and Long 1994). Overexpression of either fts $Z_{1}$ or $f t s Z_{2}$ caused branching and swelling of free-living $S$. meliloti cells, which is reminiscent of the morphologies of bacteroids (Latch and Margolin 1997). Expression of fts $Z_{1}$ and $f t s Z_{2}$ is decreased in bacteroids, as previously reported in $S$. meliloti (Becker et al. 2004). Interestingly, our transcriptome data and the results of qRT-PCR also indicate that $f t s Z_{1}$ was expressed at significantly lower levels in bacteroids, and this didn't occur in the presence of an exogenous $m r e B$ (Fig. 8). The two fts $Z$ genes are clearly not functionally redundant but require further investigations to unravel their distinct roles in free-living and symbiotic conditions. Together, our data suggest that loss of MreB in the common ancestor of Rhizobiales and a later acquisition of $f t s Z_{2}$ are the major steps for the origin and evolution of legumerhizobial symbiosis, particularly for Rhizobium, Sinorhizobium, and Mesorhizobium lineages.

\section{MATERIALS AND METHODS}

Bacterial strains, plasmids, and culture conditions.

A list of rhizobial strains and plasmids used in this study is provided in Table 1. M. huakuii 7653R and its derivative mutants were cultured in tryptone yeast $\mathrm{TY}$ medium at $28^{\circ} \mathrm{C}$, and E. coli strains were grown in Luria-Bertani medium at $37^{\circ} \mathrm{C}$ (Zhang et al. 2000). When required, antibiotics were added at the following concentrations: ampicillin at $100 \mu \mathrm{g} / \mathrm{ml}$, gentamicin at $15 \mu \mathrm{g} / \mathrm{ml}$, streptomycin at $100 \mu \mathrm{g} / \mathrm{ml}$, and kanamycin at $50 \mu \mathrm{g} / \mathrm{ml}$. Growth kinetics were examined in $200 \mu \mathrm{l}$ of TY broth with three replicates in a 96-well microtiter plate, and absorbance was measured at the wavelength of $600 \mathrm{~nm}$.

\section{Strain construction.}

A pair of primers (MreB-F and MreB-R) was designed to amplify a 1,286-bp DNA fragment containing the mreB coding
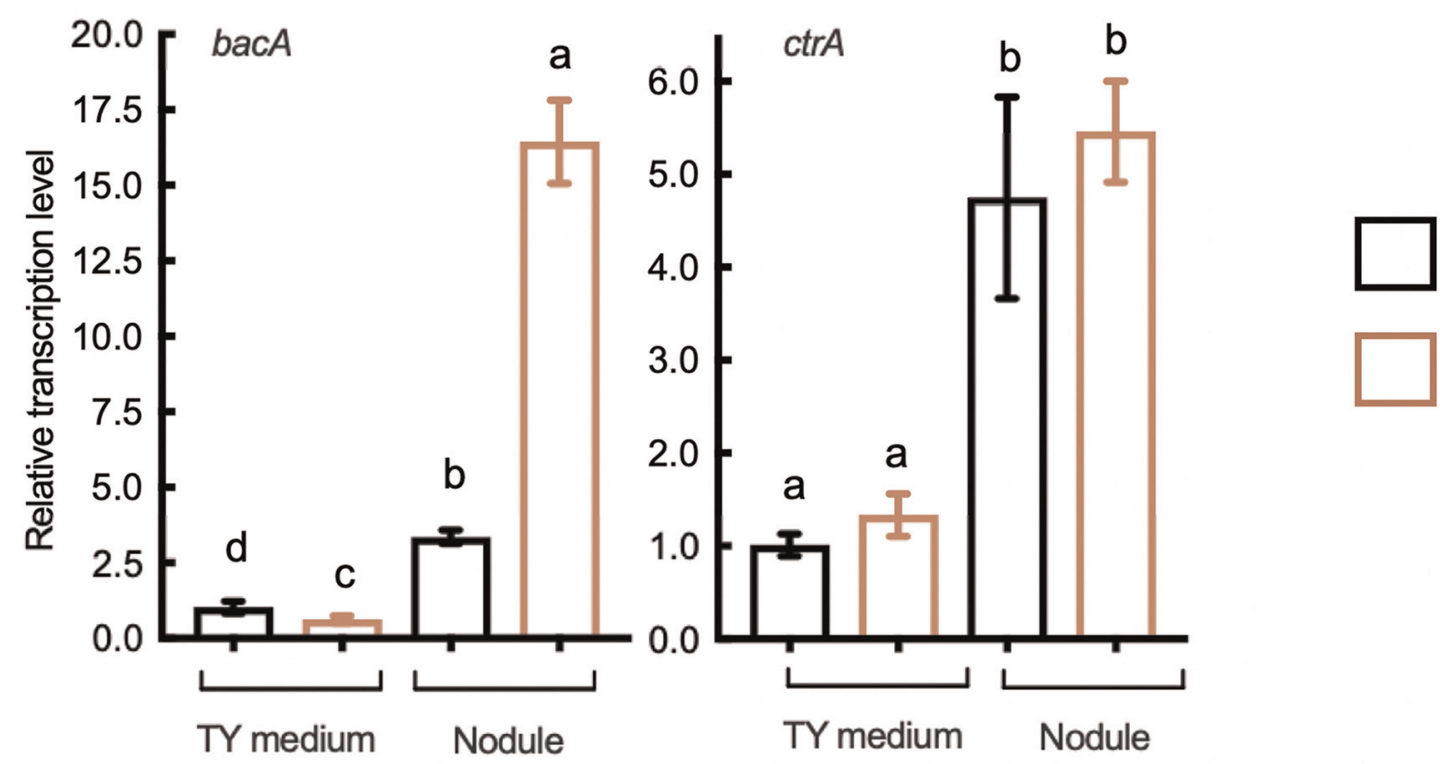

$7653 R$

\section{R-mreB}
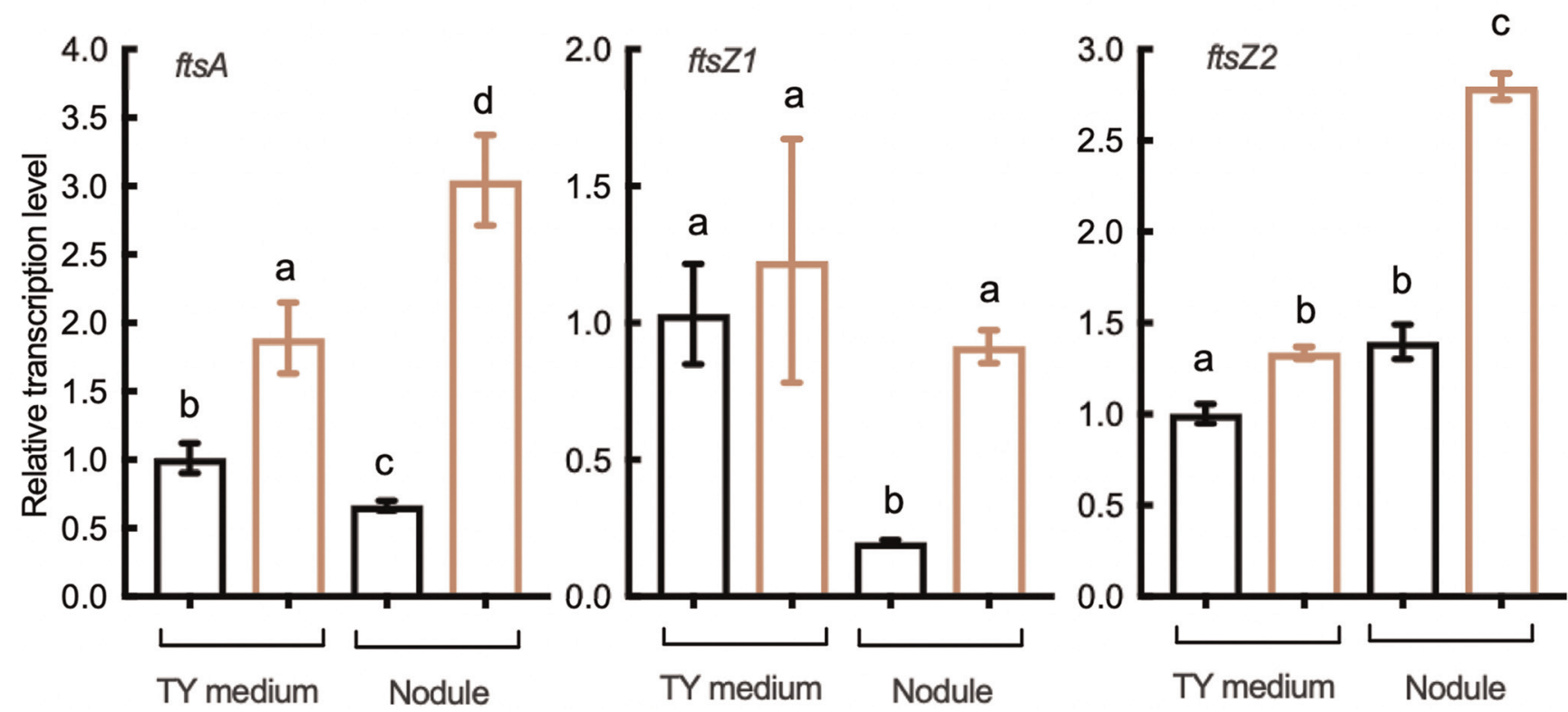

Fig. 8. Quantitative reverse-transcription PCR analysis of five differentially expressed genes identified by RNA sequencing. RNA samples were prepared from rhizobial cells grown in tryptone yeast (TY) medium at the exponential phase or nodules of Astragalus sinicus on the 28th day after inoculation. Data are means and standard errors of three biological replicates. Bars that are not connected by the same letter (shown above each) are significantly different $(P<0.05)$ by one-way analysis of variance multiple comparisons. 
region and its original promoter. PCR was performed with genomic DNA of $P$. fluorescens SBW25 as the template (Zhang and Rainey 2010). Sequences of all oligonucleotide primers used in this work are provided in Supplementary Table S7. The PCR product was first cloned into the plasmid vector pMD19T (simple) at the SpeI and HindIII sites following the manufacturer's recommendations (Takara Bio Inc.). After sequence identity was verified by DNA sequencing, the SpeI-HindIII insert DNA was subcloned into pBBR1MCS5 (Kovach et al. 1995). The resulting plasmid (pBBR5-MreB ${ }^{\mathrm{WT}}$ ) was conjugated into $M$. huakuii using the standard method of triparental mating using pRK2013 as the helper (Ditta et al. 1980).

Site-directed mutagenesis of MreB was performed with pBBR5-MreB ${ }^{\mathrm{WT}}$ using the method of splicing by overlapping extension PCR. The desired mutations were incorporated into the primers (Supplementary Table S7). The final PCR products carrying $m r e B$ mutant alleles were cloned into pBBR1MCS5 and introduced into M. huakuii 7653R as described above.

\section{Microscopy analysis.}

Standard light and electron microscopy techniques were used to visualize the cellular structure of rhizobial cells. Free-living cells were prepared by growing bacteria in the nutrient-rich TY medium to log phase, and were washed once in phosphatebuffered saline (PBS). Root nodules were fixed using formalinacetic acid-alcohol solution followed by dehydration with absolute ethanol. The nodules were then embedded in paraffin and cut longitudinally. The slices were finally stained with toluidine blue and observed under an Olympus light microscope. For liveor-dead staining, the nodule was embedded with $4 \%$ agarose and cut into $80-\mu \mathrm{M}$ thick slices using an oscillating blade microtome. Nodule slices were then treated for $15 \mathrm{~min}$ with staining buffer ( $5 \mu \mathrm{M}$ SYTO 9 and $30 \mu \mathrm{M}$ propidium iodide $[\mathrm{PI}]$ in $50 \mathrm{mM}$ Tris, $\mathrm{pH}$ 7.0) provided by the LIVE/DEAD BacLight Bacterial Viability kit (Invitrogen, Carlsbad, CA U.S.A.), followed by another 15 min of incubation with PBS buffer containing $0.01 \%$ (wt/vol) calcofluor white M2R (Sigma, Munich, Germany) to stain the plant cell wall (Nagata and Takebe 1971). The samples were then visualized under a confocal laser-scanning microscope (LSM 700; Carl Zeiss, Jena, Germany). Calcofluor has an excitation wavelength of $405 \mathrm{~nm}$ and an emission signal at 405 to $470 \mathrm{~nm}$, which are distinct from the excitation (488 and $555 \mathrm{~nm})$ and emission (490 to 522 and 555 to $700 \mathrm{~nm}$ ) wavelengths for SYTO 9 and PI, respectively. Images were captured and further analyzed using the NIS-Elements Viewer 4.50 software.

\section{Flow cytometry analysis.}

The early exponential phase cultures of 7653R and 7653R-mreB were suspended in minimal medium containing $50 \mathrm{mM} 3-(\mathrm{N}-\mathrm{mor}-$ pholino)propanesulfonic acid, $1 \mathrm{mM} \mathrm{MgSO} 4,0.25 \mathrm{mM} \mathrm{CaCl}_{2}, 19$ $\mathrm{mM}$ glutamic acid, and $0.004 \mathrm{mM}$ biotin ( $\mathrm{pH}$ 7.0). After synchronizing the cells for $12 \mathrm{~h}$, bacteria were sampled every $2 \mathrm{~h}$ over a period of $14 \mathrm{~h}$. Cells were resuspend in $1 \mathrm{ml}$ of PBS with a final cell density (absorbance at $600 \mathrm{~nm}$ ) of 0.1 , then stained with 10 $\mu \mathrm{l}$ of PI $(1 \mathrm{mg} / \mathrm{ml})$ for $30 \mathrm{~min}$ at $37^{\circ} \mathrm{C}$. Fluorescence-activated cell sorting was performed using the service provided by the Core Facility of the State Key Laboratory of Agricultural Microbiology (Wuhan, China) with a Cytoflex flow cytometer (Beckman Coulter) equipped with the FlowJo X software.

\section{Phylogenetic analysis.}

Genome assemblies (Supplementary Table S2) were retrieved from NCBI database and the FtsZ sequences were identified by BlastP search using the deduced amino acid sequences of fts $Z_{1}$ and $f$ ts $Z_{2}$ from $M$. huakuii 7653R. Multiple sequence alignment and construction of phylogenetic trees were performed in Geneious 9.1.8 (Biomatters Ltd., Auckland, New Zealand). After ClustalW alignment, sequences were trimmed and only the N-terminal conserved region (324 amino acids) was included in the neighborjoining consensus tree construction using bootstrap (1,000 replicates). Statistical analyses were performed in GraphPad Prism 7.

\section{Bacterial two-hybrid assays.}

Protein-protein interactions in vivo were assessed using the BacterioMatch II Two-Hybrid System Library Construction Kit (Stratagene), which involved a bait plasmid $\mathrm{pBT}$ and a target

Table 1. Bacterial strains and plasmids used in this work $^{\mathrm{a}}$

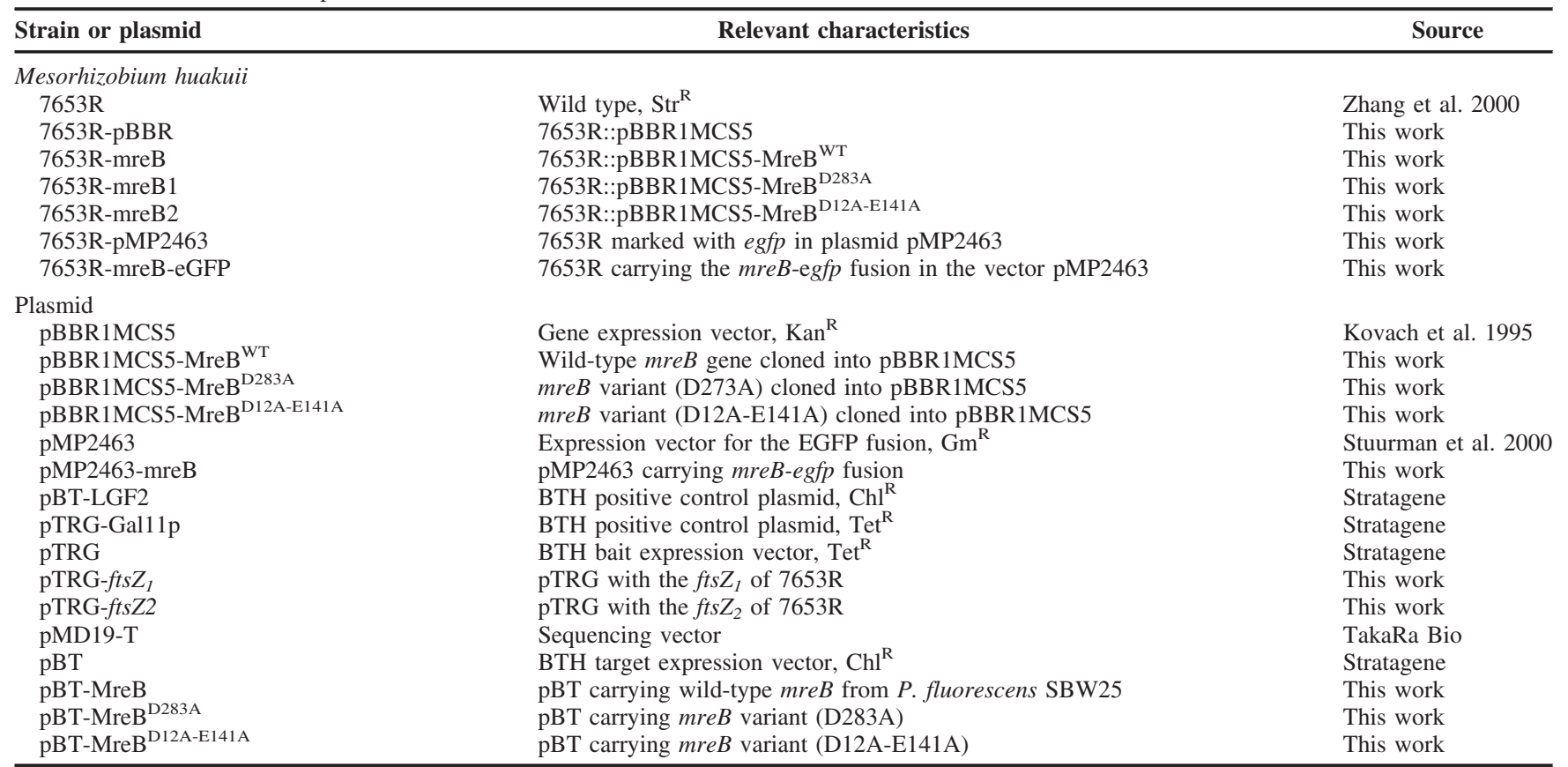

${ }^{a}$ Abbreviations: eGFP $=$ enhanced green fluorescent protein; and $\mathrm{Str}^{\mathrm{R}}, \mathrm{Kan}^{\mathrm{R}}, \mathrm{Gm}^{\mathrm{R}}, \mathrm{Chl}^{\mathrm{R}}$, and $\mathrm{Tet}^{\mathrm{R}}$ indicate resistance to streptomycin, kanamycin, gentamicin, chloramphenicol, and tetracycline, respectively. 
plasmid pTRG. Briefly, the PCR-amplified $m r e B$ or its mutant allele $(1,035 \mathrm{bp})$ was cloned into pBT at the EcoRI and BamHI sites, whereas the PCR product of $f t s Z_{1}(1,677 \mathrm{bp})$ or $f t s Z_{2}$ $(1,035 \mathrm{bp})$ was cloned into pTRG at the XhoI and BamHI sites. Next, the recombinant $\mathrm{pBT}$ and pTRG plasmids in pairs were transformed into the E. coli XL-Blue. The interaction was examined by growing the resultant bacteria $\left(24 \mathrm{~h}\right.$ at $37^{\circ} \mathrm{C}$, and then $12 \mathrm{~h}$ at room temperature) on selective screening medium supplemented with 3-amino-1,2,4-triazole, according to manufacturer instructions.

\section{Plant cultivation and symbiotic analysis.}

A. sinicus seed were surface sterilized with $1 \%$ sodium hypochlorite and $75 \%$ ethanol. After washing seven to eight times with sterile water, seed were transferred into water agar for germination (approximately 2 days at $24^{\circ} \mathrm{C}$ ). The germinated seedlings were then cultivated in pots filled with sterile sands and irrigated with the nitrogen-free Fahraeus solution. The plants were grown in chambers subjected to a daily cycle of $16 \mathrm{~h}$ of light and $8 \mathrm{~h}$ of darkness at temperatures of 22 and $20^{\circ} \mathrm{C}$, respectively. The plants were inoculated with related rhizobial strains after cotyledon expansion, and root nodules were harvested at 28 days after inoculation. Acetylene reduction (or nitrogenase) activity was measured as previously described using a gas chromatograph (model 163; Hatachi, Tokyo, Japan).

\section{RNA-seq analysis.}

The TRIzol Reagent (Aidlab Biotech, Beijing, China) was used to prepare total RNA from 7653R and 7653R-mreB cells grown in TY broth at the exponential phase and also from nodules. To isolate total RNA from plant tissues, nodules resuspended in the TRIzol reagent were first frozen in liquid nitrogen and then subjected to grinding with a mortar and pestle. All RNA samples were treated with DNaseI (Takara Bio Inc.) to remove residual genomic DNAs. RT-PCR was conducted using the Hieff qPCR SYBR Green Master Mix (High Rox Plus) (Yeasen Biotech Co., Shanghai, China). RNA-seq and analysis of the sequence data were performed on an Illumina HiSeq 4000 platform using the services of Novogene (Beijing, China).

Raw sequence data were submitted to the NCBI Sequence Reads Archive with a BioProject accession number PRJNA690069.

\section{ACKNOWLEDGMENTS}

We thank F. Wang at the State Key Laboratory of Agricultural Microbiology Core Facility for assistance with fluorescence-activated cell sorting analysis.

\section{LITERATURE CITED}

Becker, A., Bergès, H., Krol, E., Bruand, C., Rüberg, S., Capela, D., Lauber, E., Meilhoc, E., Ampe, F., de Bruijn, F. J., Fourment, J., Francez-Charlot, A., Kahn, D., Küster, H., Liebe, C., Pühler, A., Weidner, S., and Batut, J. 2004. Global changes in gene expression in Sinorhizobium meliloti 1021 under microoxic and symbiotic conditions. Mol. Plant-Microbe Interact. 17:292-303

Brown, P. J., de Pedro, M. A., Kysela, D. T., Van der Henst, C., Kim, J., De Bolle, X., Fuqua, C., and Brun, Y. V. 2012. Polar growth in the alphaproteobacterial order Rhizobiales. Proc. Natl. Acad. Sci. U.S.A. 109:1697-1701.

Caccamo, P. D., and Brun, Y. V. 2018. The molecular basis of noncanonical bacterial morphology. Trends Microbiol. 26:191-208.

Carballido-López, R. 2006. The bacterial actin-like cytoskeleton. Microbiol. Mol. Biol. Rev. 70:888-909.

Charbon, G., Cabeen, M. T., and Jacobs-Wagner, C. 2009. Bacterial intermediate filaments: In vivo assembly, organization, and dynamics of crescentin. Genes Dev. 23:1131-1144.

Coba de la Peña, T., Fedorova, E., Pueyo, J. J., and Lucas, M. M. 2018. The symbiosome: Legume and rhizobia co-evolution toward a nitrogen-fixing organelle? Front. Plant Sci. 8:2229.
Daniel, R. A., and Errington, J. 2003. Control of cell morphogenesis in bacteria: Two distinct ways to make a rod-shaped cell. Cell 113: 767-776.

De Nisco, N. J., Abo, R. P., Wu, C. M., Penterman, J., and Walker, G. C. 2014. Global analysis of cell cycle gene expression of the legume symbiont Sinorhizobium meliloti. Proc. Natl. Acad. Sci. U.S.A. 111: 3217-3224.

Ditta, G., Stanfield, S., Corbin, D., and Helinski, D. R. 1980. Broad host range DNA cloning system for gram-negative bacteria: Construction of a gene bank of Rhizobium meliloti. Proc. Natl. Acad. Sci. U.S.A. 77:7347-7351

Dye, N. A., Pincus, Z., Fisher, I. C., Shapiro, L., and Theriot, J. A. 2011. Mutations in the nucleotide binding pocket of MreB can alter cell curvature and polar morphology in Caulobacter. Mol. Microbiol. 81:368-394.

Egan, A. J. F., Errington, J., and Vollmer, W. 2020. Regulation of peptidoglycan synthesis and remodelling. Nat. Rev. Microbiol. 18:446-460.

Errington, J. 2015. Bacterial morphogenesis and the enigmatic MreB helix. Nat. Rev. Microbiol. 13:241-248.

Faguy, D. M., and Doolittle, W. F. 1998. Cytoskeletal proteins: The evolution of cell division. Curr. Biol. 8:R338-R341.

Farkas, A., Maróti, G., Durgő, H., Györgypál, Z., Lima, R. M., Medzihradszky, K. F., Kereszt, A., Mergaert, P., and Kondorosi, É. 2014 Medicago truncatula symbiotic peptide NCR247 contributes to bacteroid differentiation through multiple mechanisms. Proc. Natl. Acad. Sci. U.S.A. 111:5183-5188.

Fenton, A. K., and Gerdes, K. 2013. Direct interaction of FtsZ and MreB is required for septum synthesis and cell division in Escherichia coli. EMBO J. 32:1953-1965.

Fu, G. Y., Yu, X. Y., Zhang, C. Y., Zhao, Z., Wu, D., Su, Y., Wang, R. J., Han, S. B., Wu, M., and Sun, C. 2017. Mesorhizobium oceanicum sp. nov., isolated from deep seawater. Int. J. Syst. Evol. Microbiol. 67:2739-2745.

Haag, A. F., and Mergaert, P. 2020. Terminal bacteroid differentiation in the Medicago-Rhizobium interaction-A tug of war between plant and bacteria. Pages 600-616 in: The Model Legume Medicago truncatula. F. J. de Bruijn, ed. John Wiley \& Sons, Inc., Hoboken, NJ, U.S.A.

Kang, W., Jiang, Z., Chen, Y., Wu, F., Liu, C., Wang, H., Shi, S., and Zhang, X.-X. 2020. Plant transcriptome analysis reveals specific molecular interactions between alfalfa and its rhizobial symbionts below the species level. BMC Plant Biol. 20:293.

Kovach, M. E., Elzer, P. H., Hill, D. S., Robertson, G. T., Farris, M. A., Roop, R. M., 2nd, and Peterson, K. M. 1995. Four new derivatives of the broad-host-range cloning vector pBBR1MCS, carrying different antibiotic-resistance cassettes. Gene 166:175-176.

Kruse, T., Bork-Jensen, J., and Gerdes, K. 2005. The morphogenetic MreBCD proteins of Escherichia coli form an essential membranebound complex. Mol. Microbiol. 55:78-89.

Latch, J. N., and Margolin, W. 1997. Generation of buds, swellings, and branches instead of filaments after blocking the cell cycle of Rhizobium meliloti. J. Bacteriol. 179:2373-2381.

LeVier, K., and Walker, G. C. 2001. Genetic analysis of the Sinorhizobium meliloti BacA protein: Differential effects of mutations on phenotypes. J. Bacteriol. 183:6444-6453.

Marchetti, M., Jauneau, A., Capela, D., Remigi, P., Gris, C., Batut, J., and Masson-Boivin, C. 2014. Shaping bacterial symbiosis with legumes by experimental evolution. Mol. Plant-Microbe Interact. 27:956-964.

Margolin, W. 2009. Sculpting the bacterial cell. Curr. Biol. 19:R812-R822.

Margolin, W., and Long, S. R. 1994. Rhizobium meliloti contains a novel second homolog of the cell division gene ftsZ. J. Bacteriol. 176:2033-2043.

Masson-Boivin, C., and Sachs, J. L. 2018. Symbiotic nitrogen fixation by rhizobia-the roots of a success story. Curr. Opin. Plant Biol. 44:7-15.

Mergaert, P., Uchiumi, T., Alunni, B., Evanno, G., Cheron, A., Catrice, O., Mausset, A. E., Barloy-Hubler, F., Galibert, F., Kondorosi, A., and Kondorosi, E. 2006. Eukaryotic control on bacterial cell cycle and differentiation in the Rhizobium-legume symbiosis. Proc. Natl. Acad. Sci. U.S.A. 103:5230-5235.

Morita, Y., and Nishida, H. 2018. The common ancestor of Deinococcus species was rod-shaped. Open Bioinf. J. 11:252-258.

Nagata, T., and Takebe, I. 1971. Plating of isolated tobacco mesophyll protoplasts on agar medium. Planta 99:12-20.

Oldroyd, G. E., Murray, J. D., Poole, P. S., and Downie, J. A. 2011. The rules of engagement in the legume-rhizobial symbiosis. Annu. Rev. Genet. 45:119-144.

Peng, J., Hao, B., Liu, L., Wang, S., Ma, B., Yang, Y., Xie, F., and Li, Y. 2014. RNA-Seq and microarrays analyses reveal global differentia transcriptomes of Mesorhizobium huakuii 7653R between bacteroids and free-living cells. PLoS One 9:e93626. 
Penterman, J., Abo, R. P., De Nisco, N. J., Arnold, M. F., Longhi, R., Zanda, M., and Walker, G. C. 2014. Host plant peptides elicit a transcriptional response to control the Sinorhizobium meliloti cell cycle during symbiosis. Proc. Natl. Acad. Sci. U.S.A. 111:3561-3566.

Poole, P., Ramachandran, V., and Terpolilli, J. 2018. Rhizobia: From saprophytes to endosymbionts. Nat. Rev. Microbiol. 16:291-303.

RayChaudhuri, D., and Park, J. T. 1992. Escherichia coli cell-division gene $f t s Z$ encodes a novel GTP-binding protein. Nature 359:251-254.

Robertson, G. T., Doyle, T. B., Du, Q., Duncan, L., Mdluli, K. E., and Lynch, A. S. 2007. A Novel indole compound that inhibits Pseudomonas aeruginosa growth by targeting MreB is a substrate for MexAB-OprM. J. Bacteriol. 189:6870-6881.

Shi, H., Bratton, B. P., Gitai, Z., and Huang, K. C. 2018. How to build a bacterial cell: MreB as the foreman of E. coli construction. Cell 172: 1294-1305.

Shi, H., Colavin, A., Bigos, M., Tropini, C., Monds, R. D., and Huang, K. C. 2017. Deep phenotypic mapping of bacterial cytoskeletal mutants reveals physiological robustness to cell size. Curr. Biol. 27: 3419-3429.e4.

Shih, Y. L., and Rothfield, L. 2006. The bacterial cytoskeleton. Microbiol. Mol. Biol. Rev. 70:729-754.

Siefert, J. L., and Fox, G. E. 1998. Phylogenetic mapping of bacterial morphology. Microbiology 144:2803-2808.

Stuurman, N., Pacios Bras, C., Schlaman, H. R. M., Wijfjes, A. H. M., Bloemberg, G., and Spaink, H. P. 2000. Use of green fluorescent protein color variants expressed on stable broad-host-range vectors to visualize rhizobia interacting with plants. Mol. Plant-Microbe Interact. 13:1163-1169.

Szwedziak, P., and Löwe, J. 2013. Do the divisome and elongasome share a common evolutionary past? Curr. Opin. Microbiol. 16:745-751.

Takacs, C. N., Poggio, S., Charbon, G., Pucheault, M., Vollmer, W., and Jacobs-Wagner, C. 2010. MreB drives de novo rod morphogenesis in
Caulobacter crescentus via remodeling of the cell wall. J. Bacteriol. 192:1671-1684.

Tan, X. J., Cheng, Y., Li, Y. X., Li, Y. G., and Zhou, J. C. 2009. BacA is indispensable for successful Mesorhizobium-Astragalus symbiosis. Appl. Microbiol. Biotechnol. 84:519-526.

Typas, A., Banzhaf, M., Gross, C. A., and Vollmer, W. 2011. From the regulation of peptidoglycan synthesis to bacterial growth and morphology. Nat. Rev. Microbiol. 10:123-136.

Van de Velde, W., Zehirov, G., Szatmari, A., Debreczeny, M., Ishihara, H., Kevei, Z., Farkas, A., Mikulass, K., Nagy, A., Tiricz, H., SatiatJeunemaître, B., Alunni, B., Bourge, M., Kucho, K., Abe, M., Kereszt, A., Maroti, G., Uchiumi, T., Kondorosi, E., and Mergaert, P. 2010 Plant peptides govern terminal differentiation of bacteria in symbiosis. Science 327:1122-1126.

Wachi, M., Doi, M., Tamaki, S., Park, W., Nakajima-Iijima, S., and Matsuhashi, M. 1987. Mutant isolation and molecular cloning of mre genes, which determine cell shape, sensitivity to mecillinam, and amount of penicillin-binding proteins in Escherichia coli. J. Bacteriol. 169:4935-4940.

Wang, S., Meade, A., Lam, H. M., and Luo, H. 2020. Evolutionary timeline and genomic plasticity underlying the lifestyle diversity in Rhizobiales. mSystems 5:e00438-20.

Yulo, P. R. J., and Hendrickson, H. L. 2019. The evolution of spherical cell shape; progress and perspective. Biochem. Soc. Trans. 47:1621-1634.

Zhang, X.-X., and Rainey, P. B. 2010. Bet hedging in the underworld. Genome Biol. 11:137.

Zhang, X.-X., and Rainey, P. B. 2013. Exploring the sociobiology of pyoverdin-producing Pseudomonas. Evolution 67:3161-3174.

Zhang, X.-X., Turner, S. L., Guo, X.-W., Yang, H.-J., Debellé, F., Yang, G.-P., Dénarié, J., Young, J. P. W., and Li, F.-D. 2000. The common nodulation genes of Astragalus sinicus rhizobia are conserved despite chromosomal diversity. Appl. Environ. Microbiol. 66:2988-2995. 